\title{
Excited nucleon spectrum with two flavors of dynamical fermions
}

\author{
John M. Bulava ${ }^{a}$, Robert G. Edwards ${ }^{b}$, Eric Engelson ${ }^{*}$, Bálint Joó ${ }^{b}$, Adam Lichtl ${ }^{d}$, \\ Huey-Wen Lin ${ }^{b}$, Nilmani Mathur ${ }^{e}$, Colin Morningstar ${ }^{a}$, David G. Richards ${ }^{b}$, and \\ Stephen J. Wallace ${ }^{c}$ \\ for the Hadron Spectrum Collaboration \\ ${ }^{a}$ Department of Physics, Carnegie Mellon University, Pittsburgh, PA 15213 \\ ${ }^{b}$ Thomas Jefferson National Accelerator Facility, Newport News, VA 23606 \\ ${ }^{c}$ Department of Physics, University of Maryland, College Park, MD 20742 \\ ${ }^{d}$ RIKEN-BNL Research Center, Brookhaven National Laboratory, Upton, NY 11973 \\ ${ }^{e}$ Department of Theoretical Physics, Tata Institute of Fundamental Research, India
}

\begin{abstract}
We compute the spectrum of excited nucleons using the anisotropic Wilson lattice with two flavors of dynamical fermions. Using optimized sets of operators which transform irreducibly under the octahedral group, matrices of correlation functions are computed. We apply the variational method to these matrices to extract excited energy eigenstates. We obtain several states for each irrep and identify the continuum spin for the lowest-lying states, including a $J^{P}=\frac{5}{2}^{-}$state.
\end{abstract}

The XXVI International Symposium on Lattice Field Theory

July 14 - 19, 2008

Williamsburg, Virginia, USA

*Speaker. 


\section{Introduction}

The study of the excited baryon spectrum is an important research program for lattice QCD. In this report we present preliminary findings for the nucleon excited spectrum using the $N_{f}=2$ anisotropic Wilson action. We see evidence for a $J^{P}=\frac{5}{2}^{-}$state.

We construct operators which transform according to the irreducible representations (irreps) of the lattice rotation group (the octahedral group for cubic lattices) [1,2]. There are six doublevalued irreps: $G_{1 g}, H_{g}, G_{2 g}, G_{1 u}, H_{u}$, and $G_{2 u}$, where the "g" subscript denotes positive parity (gerade) irreps and the " $u$ " subscript denotes negative parity (ungerade) irreps. We identify the continuum limit spins by subducing the continuum rotation group to the octahedral group. The pattern of continuum spin states in each octahedral irrep is summarized in Table 1.

To extract the excited spectrum, we construct a large number of operators in each symmetry channel. It is necessary to include operators with quarks displaced with respect to one another to capture radial and orbital excitations. After selecting a manageable set of optimized operators, we use the variational method, diagonalizing the matrix of correlation functions, to extract the excited spectrum. To distinguish between scattering states and resonances, it is necessary to include multihadron operators in the matrix of correlation functions. However, we use only three quark operators in this work.

Table 1: The number of occurrences of each double valued irrep $\Lambda=\left\{G_{1}, H, G_{2}\right\}$ of the octahedral group for different values of continuum $J$ up to $\frac{11}{2}$.

\begin{tabular}{|cccccccc|}
\hline$\Lambda$ & $J=$ & $\frac{1}{2}$ & $\frac{3}{2}$ & $\frac{5}{2}$ & $\frac{7}{2}$ & $\frac{9}{2}$ & $\frac{11}{2}$ \\
\hline$G_{1}$ & & 1 & 0 & 0 & 1 & 1 & 1 \\
$H$ & & 0 & 1 & 1 & 1 & 2 & 2 \\
$G_{2}$ & & 0 & 0 & 1 & 1 & 0 & 1 \\
\hline
\end{tabular}

\section{Nucleon operators}

We construct baryon operators which transform as irreps of the octahedral group as described in [2]. The basic building blocks are covariantly displaced smeared quark fields $\left(\tilde{D}_{j}^{(p)} \tilde{\psi}(x)\right)_{A a \alpha}$ with flavor $A$, color $a$, and spin $\alpha$. The smeared quark field $\tilde{\psi}$ is displaced $p$ links in the $j$ direction ( $j=0, \pm 1, \pm 2, \pm 3$ ). Both quark smearing and gauge link smearing are necessary to reduce noise and coupling to high energy states [3]. We use Gaussian quark smearing and stout link smearing [4]. From the displaced single quark operators, we construct elemental operators

$$
\Phi_{\alpha \beta \gamma ; i j k}^{A B C}(x)=\varepsilon_{a b c}\left(\tilde{D}_{i}^{(p)} \tilde{\psi}(x)\right)_{A a \alpha}\left(\tilde{D}_{j}^{(p)} \tilde{\psi}(x)\right)_{B b \beta}\left(\tilde{D}_{k}^{(p)} \tilde{\psi}(x)\right)_{C c \gamma},
$$

where $\varepsilon_{a b c}$ is the antisymmetric Levi-Civita symbol. To construct nucleon operators we project to $I=\frac{1}{2}, I_{3}=\frac{1}{2}$ :

$$
N_{\alpha \beta \gamma ; i j k}=\Phi_{\alpha \beta \gamma ; i j k}^{u u d}-\Phi_{\alpha \beta \gamma ; i j k}^{d u u} .
$$

We use several different patterns of displacements for our three quark operators, as summarized in Table 2. Taking linear combinations of these elemental operators, we project to the irreps 
Table 2: Patterns of quark displacements employed in elemental operators. The displacement indices indicate the direction of the gauge-covariant displacement for each quark; $i, j, k=\{0, \pm 1, \pm 2, \pm 3\}$. The quarks all have the same displacement length.

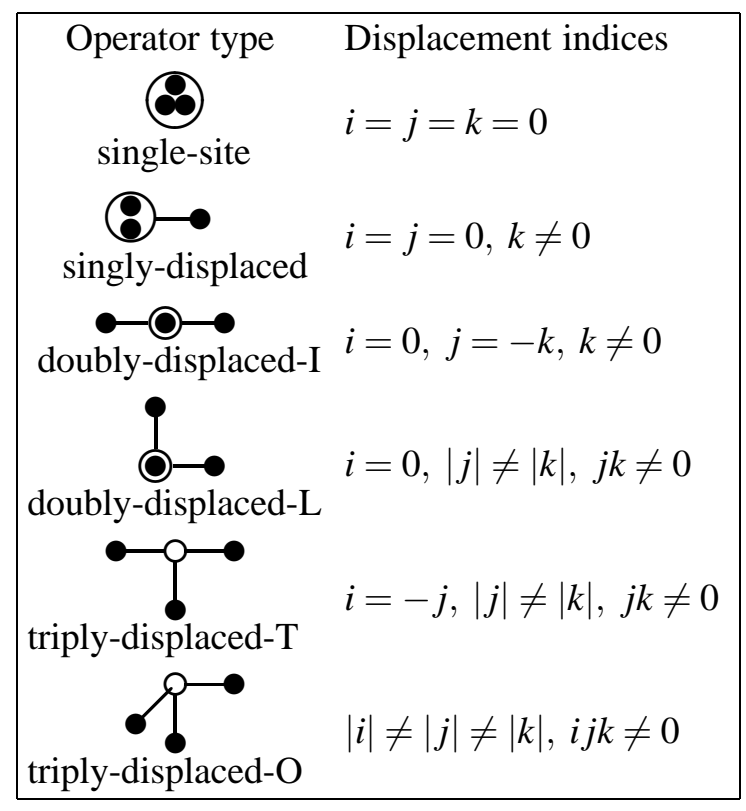

of the octahedral group. This results in hundreds of nucleon operators in each channel, which are then "pruned" to manageable sets as described in [5].

To optimize the variational method, we would like a set of low noise, linearly independent operators. We first eliminate noisy operators based on the signal to noise ratio in diagonal elements of the correlation matrix. To test for linear independence of a set of operators, we use the condition number of the normalized correlation matrix on time slice $t=a_{t}$ :

$$
\hat{C}_{i j}=\frac{C_{i j}\left(a_{t}\right)}{\sqrt{C_{i i}\left(a_{t}\right) C_{j j}\left(a_{t}\right)}} .
$$

For completely orthogonal operators, the condition number is 1 while for completely degenerate operators, the condition number diverges. We therefore seek a set of operators that minimizes the condition number. Pruning first within each operator type and then across all remaining operators, we produce a set of 16 optimal operators in each channel. The operators used in this work are the same as those in [5].

We can write the correlator of two nucleon operators in terms of three quark propagators

$$
\begin{aligned}
\tilde{G}_{(\alpha \mid \bar{\alpha})(\beta \mid \bar{\beta})(\gamma \mid \bar{\gamma})}^{(A B C)}= & \sum_{\mathbf{x}} \varepsilon_{a b c} \varepsilon_{\bar{a} \bar{b} \bar{c}} \tilde{Q}_{a \alpha \mid \bar{a} \bar{\alpha}}^{(A)}\left(\mathbf{x}, t \mid \mathbf{x}_{\mathbf{0}}, 0\right) \times Q_{b \beta \mid \bar{b} \bar{\beta}}^{(B)}\left(\mathbf{x}, t \mid \mathbf{x}_{\mathbf{0}}, 0\right) \\
& \times Q_{c \gamma \mid \bar{c} \bar{\gamma}}^{(C)}\left(\mathbf{x}, t \mid \mathbf{x}_{\mathbf{0}}, 0\right),
\end{aligned}
$$

where $Q_{a \alpha \mid \bar{a} \bar{\alpha}}^{(A)}\left(\mathbf{x}, t \mid \mathbf{x}_{\mathbf{0}}, 0\right)$ is a single quark propagator of flavor $A$ from source site $\mathbf{x}_{\mathbf{0}}$ at time $t=0$ to sink site $\mathbf{x}$ at time $t$. The nucleon correlation matrix is

$$
C_{i j}=c_{\alpha \beta \gamma}^{i} \bar{c}_{\bar{\alpha} \bar{\beta} \bar{\gamma}}^{j}\left\{\tilde{G}_{(\alpha \mid \bar{\alpha})(\beta \mid \bar{\beta})(\gamma \mid \bar{\gamma})}^{(u u d)}+G_{(\alpha \mid \bar{\beta})(\beta \mid \bar{\alpha})(\gamma \mid \bar{\gamma})}^{(u u d)}-G_{(\alpha \mid \bar{\gamma})(\beta \mid \bar{\beta})(\gamma \mid \bar{\alpha})}^{(u u d)}-G_{(\alpha \mid \bar{\beta})(\beta \mid \bar{\gamma})(\gamma \mid \bar{\alpha})}^{(u u d)}\right.
$$




$$
\left.-G_{(\beta \mid \bar{\beta})(\gamma \mid \bar{\alpha})(\alpha \mid \bar{\gamma})}^{(u u d)}-G_{(\beta \mid \bar{\alpha})(\gamma \mid \bar{\beta})(\alpha \mid \bar{\gamma})}^{(u n d)}+G_{(\gamma \mid \bar{\gamma})(\beta \mid \bar{\beta})(\alpha \mid \bar{\alpha})}^{(u n d)}+G_{(\gamma \mid \bar{\beta})(\beta \mid \bar{\gamma})(\alpha \mid \bar{\alpha})}^{(u u d)}\right\},
$$

where the $c_{\alpha \beta \gamma}^{i}$ are the coefficients to project the operators to irreps of the octahedral group. The " $i$ " superscript identifies each individual nucleon operator used to construct the matrix.

\section{Computational method}

\subsection{Variational Method}

We use the variational method $[6,5]$ to extract the excited spectrum from the matrix of correlation functions, numerically solving the generalized eigenvalue problem

$$
C_{i j}^{(\Lambda)}(t) v_{j}^{(n)}\left(t, t_{0}\right)=\alpha_{n}^{(\Lambda)}\left(t, t_{0}\right) C_{i j}^{(\Lambda)}\left(t_{0}\right) v_{j}^{(n)}\left(t, t_{0}\right),
$$

where $n$ labels the eigenstates and $\Lambda$ the double valued irrep of the octahedral group. To reduce instabilities in the eigenvectors due to degeneracies and numerical uncertainties, we solve the eigenvalue problem on a single time slice $t^{*}$. This time is selected to be as small as possible to minimize the noise, but large enough that the eigenvectors have stabilized. The correlator on all other time slices is then rotated to this fixed basis of eigenvectors.

The diagonal elements of the rotated correlation matrix $\tilde{C}_{i j}(t)$ are related to the energies by

$$
\tilde{C}_{i i}^{(\Lambda)}(t) \simeq e^{-E_{i}\left(t-t_{0}\right)}\left(1+\mathscr{O}\left(e^{-|\delta E| t}\right)\right)+\sum_{n \neq i} \alpha_{n}(t) e^{-E_{n}\left(t-t_{0}\right)},
$$

where $\delta E$ is the difference between $E_{i}$ and the next closest energy. The summation is a correction which results from the fact that we diagonalize only at $t^{*}$. On other time slices we expect a small overlap between the basis vectors and all other energy eigenstates. However, the coefficients $\alpha_{n}(t)$ should be negligible near $t^{*}$. We can calculate the fixed eigenvector effective energy via

$$
E_{i}^{e f f}(t)=\ln \left[\frac{\tilde{C}_{i i}^{(\Lambda)}(t)}{\tilde{C}_{i i}^{(\Lambda)}(t+1)}\right] .
$$

\subsection{Lattice Action}

We used $24^{3} \times 64$ anisotropic lattices with the temporal lattice spacing $a_{t}$ three times finer than the spatial lattice spacing. Gauge configurations were generated using the Wilson action. The scale was set with the Sommer parameter. We analyzed an ensemble of 430 configurations with $M_{\pi}=400 \mathrm{MeV}$.

\subsection{Fit Details}

Because baryon operators create a baryon and annihilate an antibaryon and we use antiperiodic temporal boundary conditions, if we create a baryon state at $t=0$, we also create a state which propagates backwards in time from the opposite temporal end of the lattice. Because fermions and antifermions have opposite intrinsic parity, the antibaryon propagating backward in time has parity opposite to that of the fermion propagating forward in time.

Since the signals decay exponentially, the backward-propagating signal will only be above the noise level for time slices above some threshold value of time. If the forward signal has an 
effective energy plateau in a region below this threshold then we can extract the energy of the state by fitting the correlation function with an exponential decay through the plateau without any interference from the backward propagating signal. This was, in fact, the case for all channels except for $G_{1 u}$. In this channel, the backward propagating signal was dominated by the $G_{1 g}$ ground state, the lowest energy state in the spectrum. For our lattice, the backward-propagating signal decayed slowly enough and the temporal extent was small enough (due to the anisotropy) that the $G_{1 u}$ effective energies had significant backward contamination. To extract the energy levels, we fit the correlation functions as a forward exponential decay plus a backward exponential decay which was constrained by the $G_{1 g}$ ground state.

We performed fully correlated $\chi^{2}$ minimization fits. For each state a fit range was selected such that the $\chi^{2}$ was minimized, the quality factor $Q$ was maximized, the corresponding effective energy plot plateaued in the fit range, and the fit parameters were stable under small variations in the fit range. As stated above, each channel except for the $G_{1 u}$ was modeled as

$$
\tilde{C}_{i i}^{(\Lambda)}(t)=A e^{-E_{i}^{(\Lambda)}\left(t-t_{0}\right)} .
$$

The $G_{1 u}$ correlation functions were fit simultaneously with the $G_{1 g}$ ground state, constraining the $G_{1 g}$ ground state energy to be equal to the energy of the backward-propagating $G_{1 u}$ state:

$$
\begin{aligned}
& \tilde{C}_{i i}^{\left(G_{1 u}\right)}=A e^{-E_{i}^{\left(G_{1 u}\right)}\left(t-t_{0}\right)}+B e^{-E_{0}^{\left(G_{1 g}\right)}(T-t)} \\
& \tilde{C}_{00}^{\left(G_{1 g}\right)}=D e^{-E_{0}^{\left(G_{1 g}\right)}\left(t-t_{0}\right)}
\end{aligned}
$$

We estimated the uncertainty in the energy through a jackknife analysis. We repeated the fit for each single elimination jackknife sample. The average energy and its jackknife error are reported.

\subsection{Filtering}

The presence of the backward-propagating state in the $G_{1 u}$ channel made the Lüscher method, the diagonalization of the correlation matrix on each time step, unworkable because it led to large numerical instabilities in the eigenvectors. We tested a method based on filtering out the backward signal prior to diagonalization. In a time interval where the backward signal is simply the ground state of the opposite parity channel we model the unrotated correlation matrix as

$$
C_{i j}^{(\Lambda)}(t)=\sum_{n} A_{n} e^{-E_{n}^{\Lambda}\left(t-t_{0}\right)}+B e^{-E_{0}^{\Lambda_{c}}\left(T-t_{0}\right)} .
$$

We define the filtered correlator as

$$
\begin{aligned}
C_{f i l t, i j}^{(\Lambda)}\left(t, t_{1}\right) & =C_{i j}^{(\Lambda)}(t)-C_{i j}^{(\Lambda)}\left(t_{1}\right)+\left(1-e_{0}^{-E^{\Lambda_{c}}}\right) \sum_{j=t+1}^{t_{1}} C_{i j}^{(\Lambda)}(j) \\
& =\sum_{n} A_{n}\left[1+\frac{1-e^{-E_{0}^{\Lambda_{c}}}}{e^{E_{n}^{\Lambda}}-1}\right]\left(e^{-E_{n}^{\Lambda}\left(t-t_{0}\right)}-e^{-E_{n}^{\Lambda}\left(t_{1}-t_{0}\right)}\right),
\end{aligned}
$$

where $t_{1}$ is a time where the backward signal is, in fact, described by a single exponential. The filtered correlators consist of the forward signal plus a constant term. The diagonalization of the filtered correlators using the Lüscher method produced stable eigenvectors, and the energy of the 
states could be extracted by fitting the principal correlation functions to a single exponential decay with a constant term. This method did not produce any significant improvement over the results from the fixed eigenvector method, but we point out that the filtering is necessary to extract the excited spectrum using the Lüscher method when there is significant backwards contamination in the effective energy.

\section{Results}

Four states were obtained in each channel. A plot of the spectrum is shown in Fig. 1. In the positive parity channels, we can identify the $G_{1 g}$ ground state with the nucleon. Due to the high degree of degeneracy in the excited positive parity channel, it is difficult to identify the excited states.

Results for the low energy negative-parity excited states allow for some interesting interpretations. In the $G_{1 u}$ channel, we see the energy of the lowest energy states are close to the sum of the pion and nucleon mass. This raises the possibility that one of these states is, in fact, a pion-nucleon state. Even if this is the case, the lowest negative-parity nucleon state in $G_{1 u}$ is below the first excited positive-parity state in $G_{1 g}$. This does not match the physical spectrum where the lowest negative parity resonance, the $N^{*}(1535)$ is above the first excited positive parity state, the $N(1440)$.

In the $H_{u}$ channel, we expect the lowest state to correspond to a spin $\frac{3}{2}^{-}$state. We interpret the lowest $H_{u}$ state as the $N(1520)$. In the $G_{2 u}$ channel we see that the lowest-energy is degenerate with a partner $H_{u}$ state, with no state in the $G_{1 u}$ channel at the same energy. This is the signature of a spin $\frac{5}{2}^{-}$state. Two of the six components needed for a spin $\frac{5}{2}^{-}$state are occur in the $G_{2 u}$ channel and four in the $H_{u}$ channel. The $G_{2 u}$ and $H_{u}$ states corresponding to the $\frac{5}{2}^{-}$must be degenerate in the continuum limit. Moreover, there should not be a $G_{1 u}$ state that is degenerate with these two states as that would indicate the possible presence of a spin $\frac{7}{2}^{-}$state. These conditions are satisfied near $E a_{t}=0.35$ giving clear evidence for a $\frac{5}{2}^{-}$state. We interpret this to be the lowest $\frac{5}{2}^{-}$state in the physical spectrum, the $N(1675)$.

A $\frac{5}{2}^{-}$state has not been seen in any earlier work. Our quenched QCD analysis had three degenerate states (within errors): $G_{2 u}, H_{u}$ and $G_{1 u}$. That pattern had two possible interpretations: a single spin $\frac{7}{2}^{-}$state or an accidental degeneracy of a spin $\frac{5}{2}^{-}$state and a spin $\frac{1}{2}^{-}$state.

\section{Acknowledgements}

This work was done using the Chroma software suite [7] on clusters at Jefferson Laboratory using time awarded under the USQCD Initiative. This research used resources of the National Center for Computational Sciences at Oak Ridge National Laboratory, which is supported by the Office of Science of the U.S. Department of Energy (DOE) Under contract DE-AC05-00OR22725 under the US DOE INCITE 2007 Program. This research was supported in part by the National Science Foundation through Teragrid Resources provided by the San Diego Supercomputing Center (Blue Gene). This work was also partially supported by National Science Foundation award PHY-0653315 and by the Department of Energy under contracts DE-AC05-06OR23177 and DEFG02-93ER-40762. The U.S. Government retains a non-exclusive, paid-up, irrevocable, worldwide license to publish or reproduce this manuscript for U.S. Government purposes. 


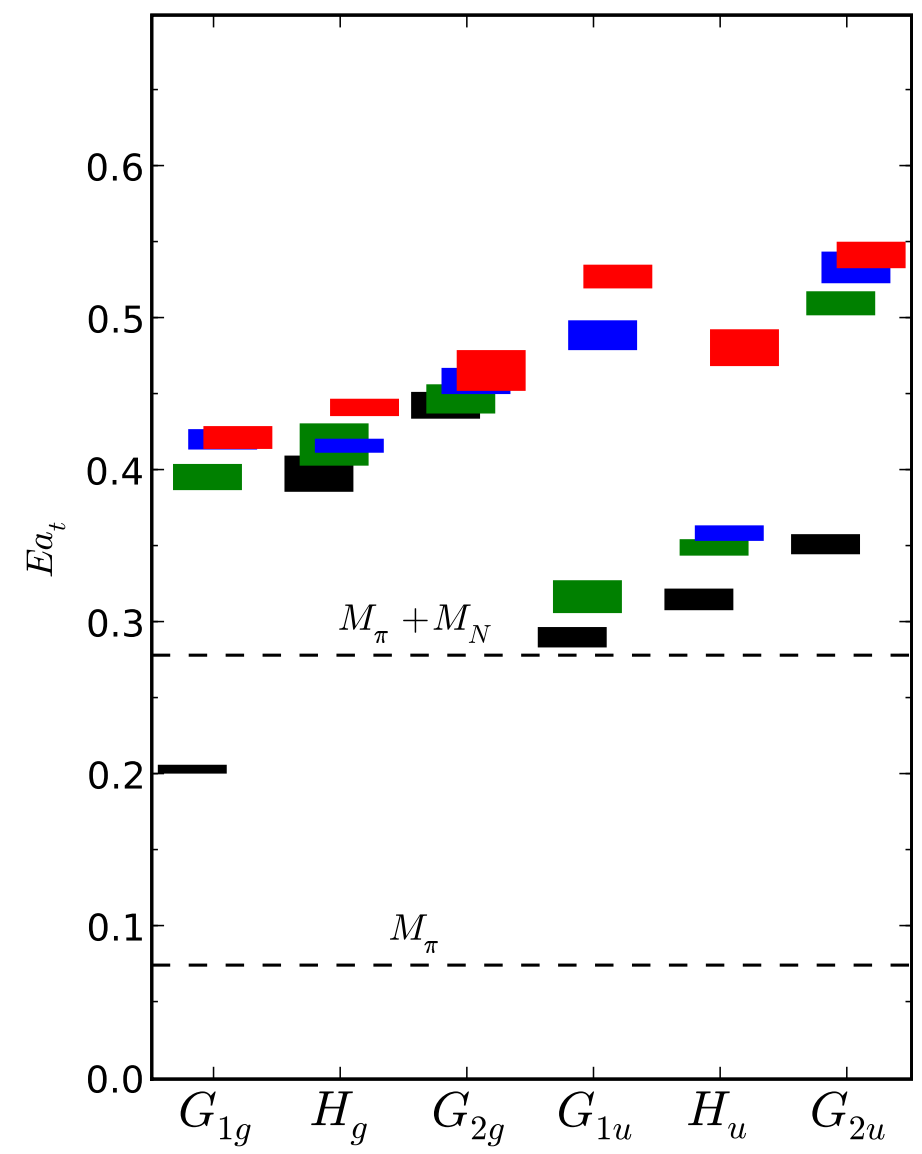

Figure 1: The nucleon spectrum obtained for each symmetry channel for $24^{3} \times 64 N_{f}=2$ lattice QCD data at $m_{\pi}=400 \mathrm{MeV}$. Errors are indicated by the vertical size of the box.

\section{References}

[1] S. Basak, et. al, Phys. Rev. D.72, 074501 [arXiv:hep-lat/0508018].

[2] S. Basak, et. al, Phys. Rev. D.72, 094506 [arXiv:hep-lat/0506029].

[3] A. Lichtl, et. al, PoS (Lat2005) 076.

[4] C. Morningstar and M. Peardon, Phys. Rev. D 69, 054501 [arXiv:hep-lat/0311018].

[5] A. Lichtl, arXiv:hep-lat/0609019.

[6] M. Lüscher and U. Wolff, Nucl. Phys. B. 339, 222.

[7] R. G. Edwards, B. Joó, Nucl. Phys. Proc. Suppl. 140, 832 [arXiv:hep-lat/0409003]. 